\title{
RESTRICTED LEFT INVERTIBLE TOEPLITZ OPERATORS ON MULTIPLY CONNECTED DOMAINS
}

\author{
KEIJI IZUCHI AND SHÛICHI OHNO \\ Dedicated to Professor Junzo Wada on his sixtieth birthday
}

\begin{abstract}
A characterization of restricted left invertible Toeplitz operators on multiply connected domains is given. To prove this, some extension theorems are given.
\end{abstract}

1. Introduction. Let $\Omega$ be a bounded connected open subset of the plane whose boundary $\Gamma$ consists of finitely many disjoint analytic Jordan curves. We denote by $H^{\infty}(\Omega)$ the space of bounded holomorphic functions on $\Omega$. In [1], Abrahamse studied Toeplitz operators on $\Omega$ and gave a left invertibility criterion for Toeplitz operators in a generalized sense. In [3], Clancey and Gosselin studied local properties of Toeplitz operators on the unit open disk and gave a characterization of restricted left invertible Toeplitz operators with the help of Younis' extension theorem [7].

In this paper, we will extend the Clancey-Gosselin-Younis theorem to multiply connected domains (§3). To prove this, we need some extension theorems as in $[7,8]$. Since $H^{\infty}(\Omega)$ is not a strongly logmodular algebra, here we cannot use the Younis extension theorems. We will give more general extension theorems $(\S 2)$.

2. Extension theorems. Let $A$ be a function algebra on $X$. Throughout this section, we assume that $X$ is a totally disconnected compact Hausdorff space. A closed subset $E$ of $X$ will be called a peak set for $A$ if there is a function $f$ in $A$, which is called a peaking function for $E$, such that $f=1$ on $E$ and $|f|<1$ on $X \backslash E$. A subset $S$ of $X$ is called a weak peak set for $A$ if it is an intersection of some peak sets. We consider the following separation condition.

For any open and closed subset $U$ of $X$, there is a function $G$ in $A$ such that $|G|>1$ on $U$ and $|G|<1$ on $X \backslash U$.

The following theorem is a generalization of [8, Theorem 3.2 and Corollary 3.3].

THEOREM 1. Suppose that $A$ satisfies separation condition (\#). Let $S$ be a weak peak set for $A$. If a function $f$ in $A$ satisfies $|f|>0$ on $S$, then there exists a function $F$ in $A$ such that $F=f$ on $S$ and $|F|>0$ on $X$.

Proof. We may assume that $f$ vanishes somewhere on $X$. Take an open and closed subset $U$ of $X$ such that $S \subset U,|f|>0$ on $U$, and

$$
|f| \leq 1 \quad \text { on } X \backslash U \text {. }
$$

Received by the editors December 9, 1985 and, in revised form, March 24, 1986.

1980 Mathematics Subject Classification (1985 Revision). Primary 30H05, 46J10, 47B35.

Key words and phrases. Restricted left invertible, a family of Toeplitz operators, function algebras. 
Put $c=\inf \{|f(x)| ; x \in U\}$. Then $c>0$. Since $S$ is a weak peak set, there is a peak set $E$ for $A$ with $S \subset E \subset U[\mathbf{5}$, p. 56]. Let $h \in A$ be a peaking function for $E$. Replacing $h$ by high powers of $h$, we may assume

$$
|h| \leq 1 / 2 \quad \text { on } X \backslash U .
$$

By our separation condition, there is a function $G$ in $A$ such that

$$
|G| \leq c / 3 \quad \text { on } U \quad \text { and } \quad|G| \geq 3 \quad \text { on } X \backslash U .
$$

Put $F=f+G(1-h)$. Clearly $F \in A$ and $F=f$ on $S$. For $x \in U$, by (3) we have

$$
|F(x)| \geq|f(x)|-|G(x)||1-h(x)| \geq c / 3>0 .
$$

For $x \in X \backslash U$, by (1), (2), and (3) we have

$$
|F(x)| \geq|G(x)||1-h(x)|-|f(x)| \geq 1 / 2>0 .
$$

Thus $|F|>0$ on $X$.

REMARK 1 . Let $A$ be a strongly logmodular algebra on a totally disconnected compact space $X$, that is, $\log \left|A^{-1}\right|=C_{R}(X)$, where $A^{-1}$ denotes the set of invertible elements in $A$ and $C_{R}(X)$ denotes the space of real continuous functions on $X$. Then $A$ satisfies (\#).

REMARK 2. For a general function algebra $A$, the assertion of Theorem 1 is not true. Put $X=\{(z, t) ; z$ is a complex number and $t$ is a real number with $|z|=1-|t|$ and $|t| \leq 1\}$. Let $A=\{g \in C(X)$; for each $t$ with $|t|<1, g(z, t)$ has a continuous holomorphic extension to $\{|z| \leq 1-|t|\}\}$. Then $S=\{(z, 0) ;|z|=1\}$ is a peak set for $A$. Put $f(z, t)=(1-|t|) z$. Then $f \in A$ and $|f|>0$ on $S$, but it is easy to see that there is no $F \in A$ with $F=f$ on $S$ and $|F|>0$ on $X$.

For a weak peak set $S$, put $A_{S}=\{f \in C(X) ; f|S \in A| S\}$. Then $A_{S}$ is a closed subalgebra of $C(X)$. For $\psi \in C(X)$ and $B \subset C(X)$, put $d(\psi, B)=\inf \{\|\psi-f\| ; f \in$ $B\}$. For $\psi \in C(X)$ and $F \subset X$, put $\|\psi\|_{F}=\sup \{|\psi(x)| ; x \in F\}$. The following theorem is a generalization of $[8$, Theorem 3.1].

THEOREM 2. Suppose that A satisfies separation condition (\#). Let $S$ be a weak peak set for $A$, and let $u$ be a function in $C(X)$ such that $\|u\|=1,|u|=1$ or $S$, and $d\left(u, A_{S}\right)<1$. Then there is a function $\tilde{u}$ in $C(X)$ such that $|\tilde{u}|=|u|$ on $X$, $\tilde{u}=u$ on $S$, and $d(\tilde{u}, A)<1$.

Proof. Since $d\left(u, A_{S}\right)<1$, there exists a function $f$ in $A$ such that $\|u-f\|_{S}<$ 1. Since $|u|=1$ on $S,|f|>0$ on $S$. By Theorem 1, there is a function $F$ in $A$ such that $F=f$ on $S$, and

$$
|F|>0 \text { on } X \text {. }
$$

Since $\|u-F\|_{S}=\|u-f\|_{S}<1$, there is an open and closed subset $U$ of $X$ such that $S \subset U$ and

$$
\|u-F\|_{U}<1 .
$$

Since $S$ is a weak peak set, there is a peak set $E$ for $A$ such that $S \subset E \subset U$. Let $h \in A$ be a peaking function for $E$. Replacing $h$ by high powers of $(1+h) / 2$, we may assume that

$$
|h|>0 \quad \text { on } X \quad \text { and } \quad 0<|h F|<1 \quad \text { on } X \backslash U .
$$


Put

$$
\tilde{u}= \begin{cases}u h /|h| & \text { on } U \\ |u| h F /|h F| & \text { on } X \backslash U .\end{cases}
$$

By $(3), \tilde{u} \in C(X)$. Clearly $|\tilde{u}|=|u|$ on $X$ and $\tilde{u}=u$ on $S$. To prove $d(\tilde{u}, A)<1$, it is sufficient to prove $\|\tilde{u}-h F\|<1$, because $h F \in A$. We have

$$
\begin{aligned}
\|\tilde{u}-h F\|_{X \backslash U} & =\left\|\frac{|u| h F}{|h F|}-\frac{h F|h F|}{|h F|}\right\|_{X \backslash U} \\
& =\||u|-|h F|\|_{X \backslash U} \\
& <1 \quad \text { by (3) and }\|u\| \leq 1 .
\end{aligned}
$$

Also we have

$$
\|\tilde{u}-h F\|_{U}=\left\|\frac{u h}{|h|}-\frac{h F|h|}{|h|}\right\|_{U}=\|u-F|h|\|_{U} .
$$

Let $x \in U$. Then

$$
\begin{aligned}
|u(x)-F(x)| h(x)|| & =|(1-|h(x)|) u(x)+| h(x)|(u(x)-F(x))| \\
& \leq 1-|h(x)|+|h(x)|\|u-F\|_{U} \quad \text { by }\|u\| \leq 1,\|h\| \leq 1 \\
& =1-|h(x)|\left\{1-\|u-F\|_{U}\right\} \\
& <1 \quad \text { by }(2) .
\end{aligned}
$$

Thus we get $\|\tilde{u}-h F\|_{U}<1$, hence $\|\tilde{u}-h F\|<1$.

If $A$ is a strongly logmodular algebra, then $A_{S}$, where $S$ is a weak peak set for $A$, is generated by $A$ and $\left\{f^{-1} ; f \in A \cap A_{S}^{-1}\right\}[2]$. Younis used this property to prove his theorem [8, Theorem 3.1]. So the following theorem is another generalization of $[8$, Theorem 3.1].

THEOREM 3. Suppose that $A$ satisfies separation condition (\#). Let $S$ be a weak peak set for $A$ such that $A_{S}$ is generated by $A$ and $\left\{f^{-1} ; f \in A \cap A_{S}^{-1}\right\}$. If $u$ is a function in $C(X)$ with $\|u\| \leq 1$ and $d\left(u, A_{S}\right)<1$, then there exists a function $\tilde{u}$ in $C(X)$ such that $|\tilde{u}|=|u|$ on $X, \tilde{u}=u$ on $S$, and $d(\tilde{u}, A)<1$.

NOTE. We do not assume $|u|=1$ on $S$.

ProOF. By our assumption, there exist functions $g$ in $A$ and $f$ in $A \cap A_{S}^{-1}$ such that

$$
\left\|u-f^{-1} g\right\|<1 \text {. }
$$

Since $f^{-1} \in A_{S},\left|f^{-1}\right|>0$ on $X$. By Theorem 1 , there is a function $F$ in $A$ such that $F=f^{-1}$ on $S$ and $|F|>0$ on $X$. Put $\tilde{u}=u f F /|f F|$. Since $|f F|>0$ on $X$, $\tilde{u} \in C(X)$. Clearly $|\tilde{u}|=|u|$ on $X$ and $\tilde{u}=u$ on $S$. To prove $d(\tilde{u}, A)<1$, suppose not. Since $\|\tilde{u}\|=\|u\| \leq 1$, we have $d(\tilde{u}, A)=1$. We note that the space of bounded linear functionals of $C(X) / A$ may be identified with $A^{\perp}$, the set of regular Borel measures $\mu$ on $X$ such that $\int_{X} \phi d \mu=0$ for every $\phi \in A$. Hence there exists $\mu \in A^{\perp}$ with the unit total variation, $\|\mu\|=1$, such that

$$
1=\int_{X} \tilde{u} d \mu=\int_{X} \frac{u f F}{|f F|} d \mu .
$$

Since $\|u\| \leq 1$, we get $((u f F) /|f F|) \mu=|\mu|$, hence

$$
u f F \mu=|f F||\mu| \text {. }
$$


Then

$$
\begin{aligned}
1 & >\left|\int_{X}\left(u-f^{-1} g\right) \frac{f F}{\|f F \mu\|} d \mu\right| & & \text { by }(1) \\
& =\frac{1}{\|f F \mu\|}\left|\int_{X} u f F d \mu\right| & & \text { by } \mu \in A^{\perp} \\
& =\frac{1}{\|f F \mu\|} \int_{X}|f F| d|\mu| & & \text { by }(2) \\
& =1 . & &
\end{aligned}
$$

This contradiction shows $d(\tilde{u}, A)<1$.

3. Restricted left invertible Toeplitz operators. Let $\Omega$ be a bounded connected open subset of the plane whose boundary $\Gamma$ consists of finitely many disjoint analytic Jordan curves. Identifying a function in $H^{\infty}(\Omega)$ with its boundary function, we may regard $H^{\infty}(\Omega)$ as an essentially supremum norm closed subalgebra of $L^{\infty}(m)$, where $m$ is the arc length measure on $\Gamma$. A closed subspace $\mathcal{M}$ of $L^{2}(m)$ with $\mathcal{M} \neq\{0\}$ is called invariant if $H^{\infty}(\Omega) \mathcal{M} \subset \mathcal{M}$, and it is called reducing if $H^{\infty}(\Omega) \mathcal{M} \subset \mathcal{M}$ and $\overline{H^{\infty}(\Omega)} \mathcal{M} \subset \mathcal{M}$. An invariant subspace of $L^{2}(m)$ is called simple if it contains no reducing subspaces. For a closed subspace $\mathcal{M}, P_{\mathcal{M}}$ denotes the orthogonal projection of $L^{2}(m)$ onto $\mathcal{M}$. For a function $\psi$ in $L^{\infty}(m)$, put $T_{\psi}^{\mathcal{M}}(f)=P_{\mathcal{M}}(\psi f)$ for every $f$ in $\mathcal{M} . \tau_{\psi}=\left\{T_{\psi}^{\mathcal{M}} ; \mathcal{M}\right.$ is a simply invariant subspace $\}$ is called a family of Toeplitz operators with a symbol $\psi$. In the case that $\Omega$ is the unit open disk, by Beurling's theorem, $T_{\psi}^{\mathcal{M}}$ is unitarily equivalent to the usual Toeplitz operator $T_{\psi}$. We call $\tau_{\psi}$ left invertible if $T_{\psi}^{\mathcal{M}}$ is a left invertible operator on $\mathcal{M}$ for every simply invariant subspace $\mathcal{M}$. In [1, Theorem 4.1], Abrahamse proved the following theorem which is a generalization of the Devinatz-Rabindranathan theorem (see [6, p. 119]).

THEOREM 4. Let $\psi$ be a function in $L^{\infty}(m)$ with $|\psi|=1$ a.e. dm. Then the following conditions are equivalent:

(i) $\tau_{\psi}$ is left invertible.

(ii) $d\left(\psi, H^{\infty}(\Omega)\right)<1$.

We note that by his proof, (i) $\Rightarrow$ (ii) is true for every $\psi \in L^{\infty}(m)$ with $\|\psi\| \leq 1$.

Let $X$ be the maximal ideal space of $L^{\infty}(m)$. Then $X$ is a totally disconnected compact Hausdorff space [4, p. 190]. Identifying a function in $L^{\infty}(m)$ with its Gelfand transform, we have $L^{\infty}(m)=C(X)$. We may consider $H^{\infty}(\Omega)$ as a function algebra on $X\left[4\right.$, p. 123]. Let $S$ be a weak peak subset of $X$ for $H^{\infty}(\Omega)$. A family of Toeplitz operators $\tau_{\psi}, \psi \in C(X)$, is called $S$-restricted left invertible if there is a function $\Psi$ in $C(X)$ such that $\Psi=\psi$ on $S$ and $\tau_{\Psi}$ is left invertible. In the case that $\Omega$ is the open unit disk, Clancey-Gosselin-Younis $[\mathbf{3}, 7]$ gave a characterization of $S$-restricted left invertible Toeplitz operators as follows: If $|\psi|=1$ a.e. $d m$, then the Toeplitz operator $T_{\psi}$ is $S$-restricted left invertible if and only if $d\left(\psi, H_{S}^{\infty}\right)<1$. We shall give a generalization of the above theorem to multiply connected domains as an application of $\S 2$. 
THEOREM 5. Let $S$ be a weak peak subset of $X$ for $H^{\infty}(\Omega)$. Let $\psi$ be a function in $C(X)$ with $|\psi|=1$ on $S$. Then the following conditions are equivalent:

(i) $\tau_{\psi}$ is $S$-restricted left invertible.

(ii) $d\left(\psi, H^{\infty}(\Omega)_{S}\right)<1$.

ProOF. (ii) $\Rightarrow$ (i) Since $X$ is totally disconnected, we may assume that $|\psi|=1$ on $X$. We note that $A=H^{\infty}(\Omega)$ satisfies separation condition (\#) in $\S 2$ [5, p. 119]. Then $H^{\infty}(\Omega), \psi$, and $S$ satisfy all assumption of Theorem 2 . Hence there exists a function $\Psi$ in $C(X)$ such that $|\Psi|=1$ on $X, \Psi=\psi$ on $S$, and $d\left(\Psi, H^{\infty}(\Omega)\right)<1$. By Theorem $4, \tau_{\Psi}$ is left invertible. So (i) holds.

(i) $\Rightarrow$ (ii) Suppose that $\tau_{\psi}$ is $S$-restricted left invertible. By our definition, there is a function $\Psi$ in $C(X)$ such that $\Psi=\psi$ on $S$ and $\tau_{\Psi}$ is left invertible. First we shall show that there is a function $h$ in $H^{\infty}(\Omega)$ such that

$$
h=1 \text { on } S, \quad\|\Psi h\| \leq 1, \quad \text { and } \quad|h|>0 \text { on } X .
$$

To prove this, put

$$
\phi=\max \{|\Psi|, 1\} .
$$

Then $\phi \in C(X), \phi \geq 1$ on $X$, and $\phi=1$ on $S$. By [5, p. 58], there is a function $g$ in $H^{\infty}(\Omega)$ such that $g=1$ on $S$ and

$$
|g| \leq 1 / \phi \text { on } X \text {. }
$$

Then $\|g\|=1$. Take a positive integer $N$ with

$$
N \geq 4 \text { and }\|\Psi\|(5 / 6)^{N} \leq 1 .
$$

Put $h=((g+2) / 3)^{N}$. Then $h \in H^{\infty}(\Omega), h=1$ on $S$, and $|h|>0$ on $X$. If $x \in X$ with $|g(x)| \leq 1 / 2$, then

$$
|\Psi(x) h(x)| \leq\|\Psi\|(5 / 6)^{N} \leq 1 \quad \text { by }(4) .
$$

If $x \in X$ with $1 / 2 \leq|g(x)| \leq 1$, then

$$
\begin{array}{rlrl}
|\Psi(x) h(x)| & \leq((|g(x)|+2) / 3)^{N}|\phi(x)| & \text { by }(2) \\
& \leq((|g(x)|+2) / 3)^{N} /|g(x)| & \text { by }(3) \\
& \leq 1 .
\end{array}
$$

The last inequality follows from $((t+2) / 3)^{N} \leq t$ for $1 / 2 \leq t \leq 1$ and $N \geq 4$. Thus $\|\Psi h\| \leq 1$ and we get (1).

We put $\Phi=\Psi h \in L^{\infty}(m)$. By $(1)$,

$$
\|\Phi\| \leq 1 \quad \text { and } \quad \Phi=\psi \text { on } S .
$$

Also we have that $\tau_{\Phi}$ is left invertible. To see this, let $\mathcal{M}$ be a simply invariant subspace of $L^{2}(m)$. Since $h \in H^{\infty}(\Omega)$ and $|h|>0$ on $X, T_{h}^{\mathcal{M}}$ is left invertible. Since $\tau_{\Psi}$ is left invertible, $T_{\Phi}^{\mathcal{M}}=T_{\Psi}^{\mathcal{M}} T_{h}^{\mathcal{M}}$ is left invertible, so $\tau_{\Phi}$ is left invertible. As noted after Theorem 4, we get $d\left(\Phi, H^{\infty}(\Omega)\right)<\|\Phi\| \leq 1$. Hence

$$
\begin{aligned}
d\left(\psi, H^{\infty}(\Omega)_{S}\right) & =d\left(\Phi, H^{\infty}(\Omega)_{S}\right) \quad \text { by }(5) \\
& \leq d\left(\Phi, H^{\infty}(\Omega)\right)<1
\end{aligned}
$$

This completes the proof. 


\section{REFERENCES}

1. M. B. Abrahamse, Toeplitz operators in multiply connected regions, Amer. J. Math. 96 (1974), 261-297.

2. S. Axler, Subalgebras of $L^{\infty}$, Thesis, University of California, Berkeley, 1975.

3. K. Clancey and J. Gosselin, On the local theory of Toeplitz operators, Illinois J. Math. 22 (1978), 449-458.

4. S. Fisher, Function theory on plane domains, Wiley, New York, 1983.

5. T. W. Gamelin, Uniform algebras, Prentice-Hall, Englewood Cliffs, N.J., 1969.

6. D. Sarason, Function theory on the unit circle, Lecture Notes, Virginia Polytech Inst. and State Univ., Blacksburg, Virginia, 1978.

7. R. Younis, Eatension results in the Hardy space associated with a logmodular algebra, J. Funct. Anal. 39 (1980), 16-22.

'8. _ Interpolation in strongly logmodular algebras, Pacific J. Math. 102 (1982), 247-251.

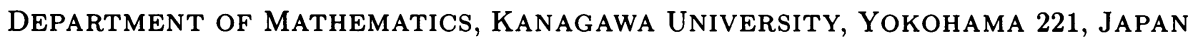

